\title{
Plethysmographic and Biochemical Markers in the Diagnosis of Endothelial Dysfunction in Pediatric Acute Lymphoblastic Leukemia Survivors - New Applications
}

\author{
A. MASOPUSTOVÁ ${ }^{1}$, P. JEHLIČKA ${ }^{1}$, M. HUML ${ }^{1}$, T. VOTAVA ${ }^{1}$, L. TREFIL $^{2}$, \\ M. KRESLOVÁ ${ }^{1}$, J. SÝKORA ${ }^{1}$
}

${ }^{1}$ Department of Pediatrics, Faculty of Medicine in Pilsen, Faculty Hospital, Charles University in Prague, Pilsen, Czech Republic, ${ }^{2}$ Department of Clinical Biochemistry and Hematology, Faculty of Medicine in Pilsen, Faculty Hospital, Charles University in Prague, Pilsen, Czech Republic

Received August 28, 2017

Accepted January 26, 2018

On-line September 11, 2018

\section{Summary}

Acute lymphoblastic leukemia (ALL) and its treatment are associated with endothelial dysfunction (ED) and increased cardiovascular risk in adulthood. There are no data on ED in children after successful treatment of ALL. We aimed to assess new ED in these children using the plethysmographic reactive hyperemia index (RHI) and biomarkers that are known to be related to ED. In all, 22 children (mean 15.6 years), after successful treatment of ALL, and 18 healthy subjects were included in this prospective study. $\mathrm{RHI}$, plasma concentrations of asymmetric dimethyl arginine (ADMA), high-sensitive CRP (hsCRP) and E-selectin were measured in all children. RHI values were significantly lower in ALL patients when compared with healthy controls $(p<0.05)$. hsCRP was significantly increased in ALL patients compared with the control group $(p<0.001)$. E-selectin plasma levels were higher in ALL patients as compared to healthy controls $(p=0.05)$. This is the first study that combines both plethysmographic and biochemical methods to assess ED in ALL survivors. Significantly decreased RHI with elevated plasma concentrations of biochemical markers imply a possible association with premature ED in ALL patients. The combined diagnostic approach seems to be a valuable tool for more accurate detection of ED and preventive cardiovascular management in these patients.

\section{Key words}

Endothelial dysfunction • Reactive hyperemia index • E-selectin • Acute lymphoblastic leukemia • Children

\section{Corresponding author}

A. Masopustová, Department of Pediatrics, Faculty of Medicine in Pilsen, Faculty Hospital, Charles University in Prague, Alej Svobody 80, 304 00, Pilsen, Czech Republic. Fax: +420 377104694. E-mail: masopustovaa@fnplzen.cz

\section{Introduction}

Acute lymphoblastic leukemia represents one fourth of all childhood malignancies (Gurney et al. 1995). The continuously improving cure rates of ALL exceed $85 \%$ nowadays (Horner et al. 2009). However, this success come hand-in-hand with increasing numbers of late adverse effects (Silverman 2014, Oeffinger et al. 2006). There is a growing population of pediatric ALL survivors at risk for developing late complications of their chemotherapy and radiotherapy given years or decades earlier (Bottomley and Kassner 2003, De Caro et al. 2011, Leger et al. 2015, Mody et al. 2008, KadaLottick et al. 2010, Bhatia 2003). Accordingly, extended life-long medical care for all survivors of ALL is warranted (Nathan et al. 2005, Essig et al. 2014). Anhracycline-induced cardiotoxicity may lead to heart failure and dilated cardiomyopathy in children (Bryant et al. 2007). In this regard, anthracyclines may also induce changes in the vascular endothelium and could be a causative agent related to the pathogenesis of ED and premature atherosclerosis in young cancer survivors (Woo et al. 2013, Jarvela et al. 2013, Barry et al. 2007). 
ED might be regarded as an integrated index of all atherogenic and atheroprotective factors present in an individual, including known, as well as yet-unknown, variables and genetic predispositions (Bonneti et al. 2003, Morisson et al. 2010). Altered endothelial cell biology is thought to be part of the early pathogenesis of atherosclerosis, although the precise mechanism is yet to be discovered. The decreased bioactivity of nitric oxide (NO), leading to the promotion of vasoconstriction, inflammation and thrombosis, plays a crucial role in the pathogenesis of ED. From a clinical point of view, ED is characterized by reduced vasodilative responses to an appropriate ischemic stimulus (Hamburg et al. 2008).

The reversibility of ED may be an attractive primary target in the effort to optimize therapeutic strategies and to decrease cardiovascular risk in pediatric ALL survivors. Early detection of this process may have therapeutic and prognostic implications (Widlanski et al. 2003). Unfortunately, ED has received little attention from researchers and studies on ED incidence in comparable pediatric ALL populations are lacking. Expanding on this, novel non-invasive diagnostic tools ensuring the capture of ED are needed (Bonneti et al. 2003). Based on these premises, the current study was conducted with the following specific aims: 1) to determine whether a significant difference in reactive hyperemia index (RHI) is found in pediatric ALL survivors as compared to controls, 2) to discern if the association between RHI and specific biochemical markers in ALL survivors exists, and 3) to demonstrate whether the combination of RHI and biochemical parameters can be used for the detection of ED in pediatric ALL survivors.

\section{Materials and Methods}

The ALL group included 22 ALL survivors at least two years after successful cytostatic therapy. All of these subjects were treated according to either BFM ALL 95 or ALL IC-BFM 2002 protocols. Neither patients with relapsed ALL nor with secondary ALL were enrolled in the study. All subjects had undergone a standard cardiology examination and all subjects had normal both systolic and diastolic heart function throughout the follow-up. Treatment protocols involved a combination of cytostatic drugs, including anthracycline chemotherapy agents (daunorubicine, doxorubicine) and cyclophosphamide. Cumulative doses of anthracyclines and cyclophosphamide were 240-360 $\mathrm{mg} / \mathrm{m}^{2}$ and $3,000 \mathrm{mg} / \mathrm{m}^{2}$, respectively.

In the control group, 18 healthy subjects without a history of inflammatory, metabolic or neoplastic diseases were enrolled. None of them reported a history of heart disease, treatment with antibiotics, antiinflammatory drugs or medication with known adverse effects on the endothelial function. Demographic characteristics of the study sample are listed in Table 1. Exclusion criteria for both groups were dyslipidemia, positive family history of premature cardiovascular events, abnormal left-ventricular function, and smoking.

Table 1. Demographic and laboratory characteristics of the study sample.

\begin{tabular}{lccc}
\hline Data & ALL & Controls & P value \\
\hline Subjects & 22 & 18 \\
Gender (boys/girls) & $15 / 7$ & $5 / 13$ & \\
Age $($ years $)$ & $15.6(12.72 ; 17.95)$ & $16.1(12.91 ; 17.33)$ & $\mathrm{NS}$ \\
BMI $\left(\mathrm{kg} / \mathrm{m}^{2}\right)$ & $21.1(19.3 ; 25.13)$ & $19.97(18.79 ; 22.85)$ & $\mathrm{NS}$ \\
Arterial blood pressure $(\mathrm{mm} \mathrm{Hg})$ & $116(105 ; 121) / 64(60 ; 70)$ & $114.5(110 ; 119) / 62.5(59 ; 69.5)$ & $\mathrm{NS}$ \\
Reactive hyperemia index $(\mathrm{RHI})$ & $1.5(1.3 ; 2.0)$ & $1.80(1.59 ; 2.46)$ & $\mathrm{p}<0.05$ \\
E-selectin $(\mu \mathrm{g} / \mathrm{l})$ & $76(58.32 ; 108.98)$ & $62.5(31.66 ; 70.99)$ & $\mathrm{p}=0.05$ \\
ADMA $(\mu \mathrm{mol} / \mathrm{l})$ & $0.6(0.53 ; 0.66)$ & $0.58(0.49 ; 0.61)$ & $\mathrm{NS}$ \\
hSCRP $($ mg/l) & $1.1(0.71 ; 2.29)$ & $0.19(0.18 ; 0.45)$ & $\mathrm{p}<0.001$ \\
VCAM $(\mu \mathrm{g} / \mathrm{l})$ & $941.7(818.66 ; 1074.0)$ & $918.4(793.08 ; 1017.90)$ & $\mathrm{NS}$ \\
Total cholesterol $($ mmol/l) & $4.2(3.77 ; 4.67)$ & $4.32(3.93 ; 4.77)$ & $\mathrm{NS}$ \\
\hline
\end{tabular}

Values are expressed as median with inter-quartile range. ADMA - asymmetric dimethyl arginine, VCAM - vascular cell adhesive molecule, hsCRP - high sensitive C-reactive protein, BMI - body mass index, ALL - acute lymphoblastic leukemia, NS - non significant. 
Reactive hyperemia index measurement

An EndoPAT ${ }^{\circledR}$ recorder (Itamar Caesarea ${ }^{\circledR}$, Israel) was used for the determination of the RHI by measuring post-occlusive endothelium-dependent changes in vascular tone (PAT) in the subject's fingertips. Initially, body mass index (BMI) and blood pressure in the non-dominant arm were measured. The subject was asked to rest in the supine position for $15 \mathrm{~min}$ in a temperature-controlled, quiet room with the unique biosensors placed on the fingertips. Then followed a five-minute brachial occlusion $(60 \mathrm{~mm} \mathrm{Hg}$ above the systolic pressure, at least $200 \mathrm{~mm} \mathrm{Hg}$ ). The postocclusive dilatation with a reactive hyperemia was captured as an increase of the PAT signal amplitude. The RHI was calculated automatically thereafter. The contralateral arm was measured simultaneously to eliminate endothelium-independent changes in vascular tone.

\section{Laboratory measurements}

We obtained blood samples from all participating children in standard clinical settings after an overnight fasting. Specific biochemical markers of ED were: asymmetric dimethyl arginine (ADMA) as a competitive NO-synthase inhibitor, E-selectin and vascular cell adhesive molecules (VCAM) measured with Elisa (OLD Diagnostica ${ }^{\circledR}$, BioVendor ${ }^{\circledR}$ ), high-sensitive CRP (hsCRP) measured by a particle-enhanced immunoturbidimetric assay technique (Orion Diagnostica ${ }^{\circledR}$ ). All samples were collected by a qualified person and the tests were performed in a blinded fashion.

\section{Statistics}

Values obtained from individual measurements are expressed using descriptive statistics (mean, standard deviation, median, inter-quartile range). Statistical differences between healthy controls and patients with ALL were calculated using a sum rank test (MannWhitney test). All statistical analyses were performed using Software SAS 9.4 (Cary ${ }^{\circledR}$, NC, USA). For all analyses, a p-value of 0.05 or less was accepted as being statistically significant.

\section{Ethical considerations}

Our study was conducted in accordance with the principles of the Declaration of Helsinki. The local Ethics Committee approved the study protocol. An informed consent was obtained from the parents or legal guardians of all enrolled participant. The research was not sponsored by any company.

\section{Results}

\section{Demographics of the study sample}

A total of 40 children were eligible for inclusion. Refer to Table 1 for an overview of the baseline demographic and clinical characteristics of both the ALL survivors and the healthy controls (HC). Probands were of Caucasian ethnicity, comparable in regard to number ratio. There were no differences in lipid profile between the study groups.

\section{Reactive hyperemia index and biochemical parameters}

RHI was significantly lower in the ALL patients as compared to $\mathrm{HC}$ : $1.5(1.3 ; 2.0), 1.8$ (1.59; 2.46), median and interquartile range, respectively; $\mathrm{p}<0.05$ (Fig. 1).

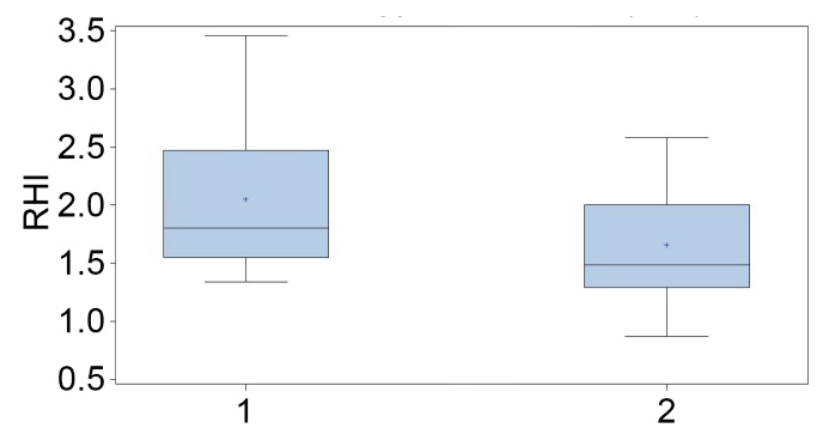

Fig. 1. RHI in healthy controls and ALL. Boxes indicate the interquartile range. Horizontal lines within boxes indicate medians. Whiskers extend to the highest or lowest values. 1 - healthy controls, 2 - acute lymphoblastic leukemia.

hsCRP plasma levels were significantly higher in ALL patients as compared to $\mathrm{HC}: 1.1(0.71$; $2.29) \mathrm{mg} / \mathrm{l} ; 0.19(0.18 ; 0.45) \mathrm{mg} / \mathrm{l}$; respectively; $\mathrm{p}<0.001$ (Fig. 2).

E-selectin levels of the children with ALL were higher when compared to HC: $76.0(58.32 ; 108.98) \mu \mathrm{g} / \mathrm{l}$, 62.5 (31.66; 70.99) $\mu \mathrm{g} / \mathrm{l}$; respectively; $\mathrm{p}=0.05$ (Fig. 2).

No significant difference in ADMA levels was observed between ALL patients and the HC 0.60 (0.53; $0.66) \mu \mathrm{mol} / \mathrm{l} ; \quad 0.58 \quad(0.49 ; \quad 0.61) \mu \mathrm{mol} / 1, \quad$ respectively (Fig. 2).

No significant difference in VCAM plasma levels was observed between ALL patients and the HC: $941.7(818.65 ; 1074.0) \mu \mathrm{g} / 1,918.4$ (793.08; 1017.90) $\mu \mathrm{g} / \mathrm{l}$; respectively (Fig. 2). 

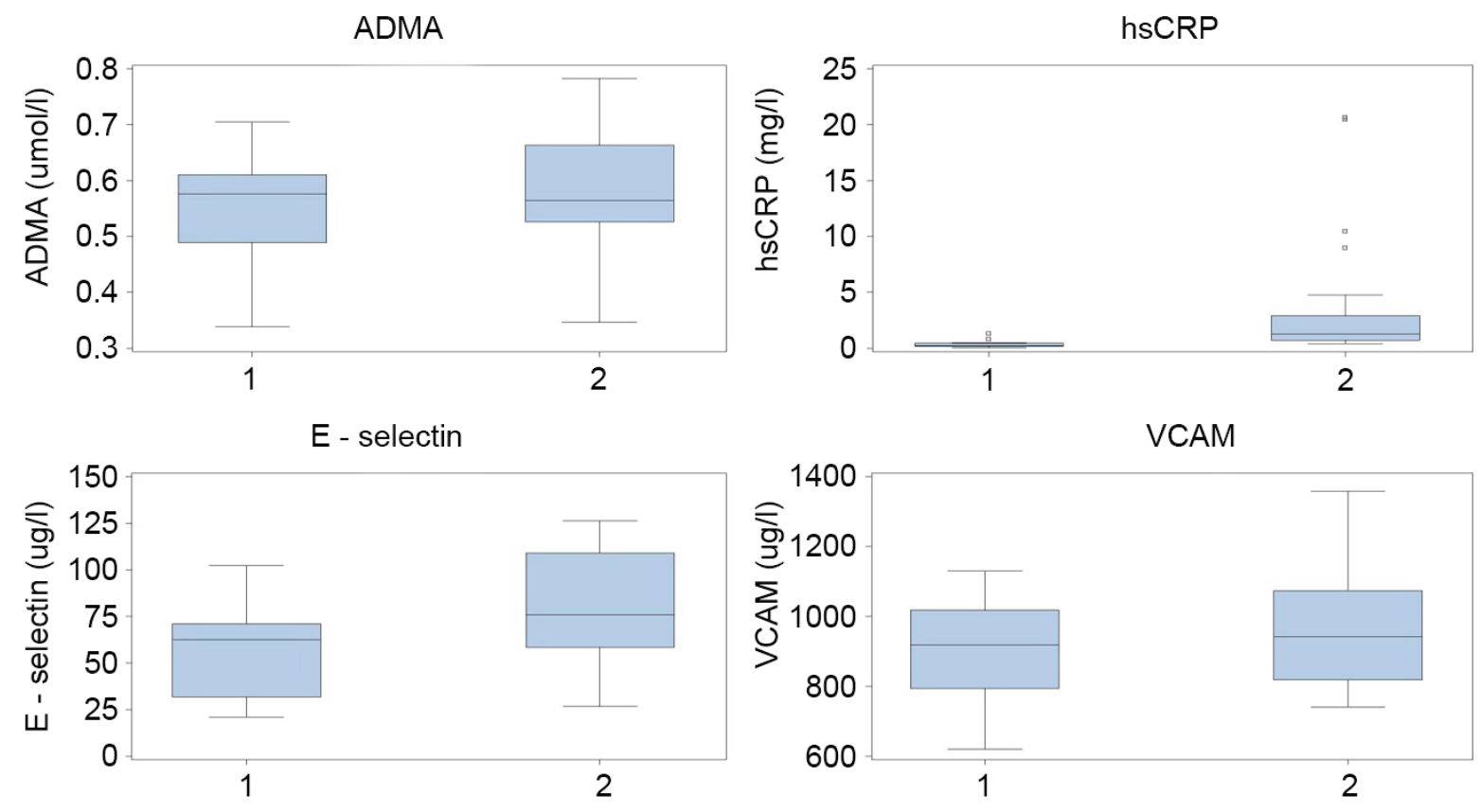

Fig. 2. Biochemical markers in healthy controls and ALL. Boxes indicate the inter-quartile range. Horizontal lines within boxes indicate medians. Whiskers extend to the highest or lowest values. 1 - healthy controls, 2 - acute lymphoblastic leukemia.

\section{Discussion}

Our study is the first to comprehensively evaluate ED in pediatric ALL survivors with respect to biochemical markers and RHI. Results of the study indicate that this combined approach may be used for the detection of ED in pediatric ALL survivors. There are several innovative findings in this study: 1) significantly reduced RHI and 2) elevated plasma levels of biochemical markers of ED when compared to healthy controls.

Several studies confirmed an increased cardiovascular risk in adult ALL survivors. The sequelae include predominantly well-documented components of metabolic syndrome with obesity, hypertension, insulin resistance and diabetes that increase cardiovascular risk (Alberti et al. 2006). Reported experience regarding ED and risk of cardiovascular diseases in young ALL survivors is limited. Only two studies implicated ED as marker of early development of atherosclerosis in ALL survivors in childhood (Woo et al. 2013, Jarvela et al. 2013). Probands in our study have a normal lipid profile and absence of metabolic syndrome (Table 1). Based on this fact, the association between treatment-related changes and ED seems to be plausible. Significantly elevated hsCRP level in children with ALL is in accordance with the proven relationship of hsCRP to atherogenic processes. Its elevated level is a predictive factor of morbidity and mortality from cardiovascular events in apparently healthy individuals, regardless of conventional risk factors (Wilson et al. 2008). One should respect that high plasmatic levels of hsCRP may be associated with acute inflammatory changes instead ED. Elevated E-selectin level reflects endothelial activation. ADMA as a competitive NO-synthase inhibitor is related to increased oxidative stress and postocclusive vasodilation. The association of significantly elevated ADMA levels and ED was confirmed in our previous studies involving children with type 1 diabetes mellitus, familial hypercholesterolemia and Crohn's disease as well (Jehlicka et al. 2014, Jehlicka et al. 2009). This relationship is supported by experimental studies confirming an intimate link among biochemical and functional endothelial changes (Mori-Kawabe et al. 2015). Surprisingly, we observed only slightly elevated ADMA plasma levels in ALL patients compared to healthy controls.

In respect to cardiovascular prevention, most of the published studies focused on cardiotoxicity of anthracyclines and related cardioprotection. Dexrazoxan, carvedilol or various alimentary supplements, such as L-carnitine, coenzyme Q and others, were assessed in many studies that ultimately had ambiguous results (Jarvela et al. 2013, Van Dalen et al. 2011). No valuable information regarding the effect of cardioprotective agents to endothelial functions in ALL survivors has been 
published to date. Only one study confirmed the positive effect of home-based exercise on endothelial function in children ALL survivors (Jarvela et al. 2013). From this point of view, further studies are highly needed.

From a technical aspect, many studies focused on ED used ultrasound methods, such as flow mediated dilatation (FMD) or intima media thickness (IMT). Reproducibility and accuracy of FMD are still matters of debate in children, mostly due to high dependency on operator's experience and demanding technical requirements (Al-Qaisi et al. 2008). IMT gradually increases during childhood, thus the precise cut-off values remain difficult to set in this population (Touboul et al. 2012). RHI, as a plethysmographic method, is technically easy to perform. Automatic analysis substantially reduces operator dependency and simultaneous measurement of the contralateral non-occluded arm allows for the elimination of unintended exogenous changes during examination. The close relationship between decreased RHI and coronary dysfunction was confirmed via invasive methods (Selamet et al. 2009). Osika et al. (2011) demonstrated a satisfactory variation coefficient of RHI and no sex differences in RHI score in a study including 248 school children.

Several limitations of the study merit consideration. One weakness of this study is the lack of a precise cut-off limit for RHI in children. Another limitation is that the sensors are unified and the minimum finger thickness is not defined. To avoid inaccurate results caused by measuring younger children with fingers too small for sensors, we only selected participants of age 12 and higher. Kelly et al. (2014) demonstrated that younger age is associated with lower RHI but not lower FMD among children and adolescents and results of their study suggest that age is associated with RHI. Therefore, we selected the control group with age comparable to ALL survivors. Hamburg et al. (2008) demonstrated increased baseline pulse amplitude (PAT) in obese persons with metabolic syndrome and an inverse relationship between baseline and response PAT to hyperemia. Some studies did not find a correlation between FMD and RHI with respect to ED (Allan et al. 2013). This controversial finding might partly be explained by different properties of large conductive brachial arteries and the peripheral resistive artery bed. The precise underlying condition is still unclear and a definitive explanation still remains an area of research. There were published conflicting results regarding the ability of RHI to detect acute alterations of vasodilatation following smoking in a small study by Moerland et al. (2012). However, smokers were not enrolled in the current sample.

\section{Conclusions}

Plethysmographic RHI and biochemical markers were used to assess ED in young ALL survivors in this study. Significantly decreased RHI, elevated plasma levels of hsCRP and E-selectin support a hypothesis of increased risk of premature ED in these patients. The combined approach seems to be a promising method for the assessment of endothelial function. Further clinical ED investigations providing data for diagnostic and therapeutic applications are needed. To what extent it will influence the late cardiovascular risk remains to be answered by long-term studies.

\section{Conflict of Interest}

There is no conflict of interest.

\section{Acknowledgements}

This study was supported by research grants MSM-0021620819, PRVOUK P-36 and PROGRES Q39.

\footnotetext{
Abbreviations

ADMA - asymmetric dimethyl arginine, ALL - acute lymphoblastic leukemia, ED - endothelial dysfunction, FMD - flow-mediated dilatation, hsCRP - high-sensitive C-reactive protein, IMT - intima-media thickness, NO nitric oxide, PAT- peripheral arterial tone, RHI - reactive hyperemia index, VCAM - vascular cell adhesive molecule.
}

\section{References}

ALBERTI KG, ZIMMET P, SHAW J: Metabolic syndrome - a new world-wide definition. A consensus statement from the International Diabetes Federation. Diabet Med 23: 469-480, 2006. 
ALLAN RB, DELANEY CL, MILLER MD, SPARK JI: A comparison of flow-mediated dilatation and peripheral artery tonometry for measurment of endothelial function in healthy individuals and patents with peripheral artrial disease. Eur J Vasc Endovasc Surg 45: 263-269, 2013.

AL-QAISI M, KHARBANDA RK, MITTAL TK, DONALD AE: Measurment of endothelial function and its clinical utility for cardiovascular risk. Vasc Health Risk Manag 4: 647-652, 2008.

BARRY E, ALVAREZ JA, SCULLY RE, MILLER TL, LIPSHUTZ SE: Anthracycline-induced cardiotoxicity: course, pathophysiology, prevention and management. Expert Opin Pharmacother 8: 1039-1058, 2007.

BHATIA S: Late effects among survivors of leukemia during childhood and adolescence. Blood Cells Mol Dis 31: 84-92, 2003.

BONNETI PO, LERMAN LO, LERMAN A: Endothelial dysfunction - a marker of atherosclerotic risk. Arterioscler Thromb Vasc Bio 23: 168-175, 2003.

BOTTOMLEY SJ, KASSNER E: Late effects of childhood cancer therapy. J Pediatr Nurs 18: 126-133, 2003.

BRYANT J, PICOT J, LEVITT G, SULLIVAN I, BAXTER L, CLEGG A: Cardioprotection against the toxic effects of anthracycline given to children with cancer: a systematic review. Health Technol Assess 11: 1-84, 2007.

DE CARO E, SMERALDI A, TROCCHIO G, CALEVO M, HANAU G, PONGIGLIONE G: Subclinical cardiac dysfunction and exercise performance in childhood cancer survivors. Pediatr Blood Cancer 56: 122-126, 2011.

ESSIG S, LI Q, CHEN Y, HITZLER J, LEISENRING W, GREENBERG M, SKLAR C, HUDSON MM, ARMSTRONG GT, KRULL KR, NEGLIA JP, OEFFINGER KC, ROBISON LL, KUEHNI CE, YASUI Y, NATHAN PC: Risk of late effects of tratment in children with newly diagnosed with standard-risk acute lymphoblastic leukemia: a report from the Childhood Cancer Survivors Study cohort. Lancet Oncol 15: 841-851, 2014.

GURNEY JG, SEVERSON RK, DAVIS S, ROBISON LL: Incidence of cancer in children in the United States. Sex-, race-, and 1-year age specific rates by histologic type. Cancer 75: 2186-2195, 1995.

HAMBURG NM, KEYES MJ, LARSON MG, VASAN RS, SCHNABEL R, PRYDE MM, MITCHELL GF, SCHEFFY J, VITA JA, BENJAMIN EJ: Cross-sectional relations of digital vascular function to cardiovascular risk factors in the Framingham Heart Study. Circulation 117: 2467-2474, 2008.

HORNER MJ, RIES LAG, KRAPCHO M, NEYMAN N, AMINOU R, HOWLADER N, ET AL.: SEER Cancer Statistics Rewiew, 1975-2006. National Cancer Institute, Bethesda, MD, 2009. https://seer.cancer.gov/archive/csr/1975_2006/index.html

JARVELA LS, NIINIKOSKI H, HEINONEN OJ, LAHTEENMAKI PM, AROLA M, KEMPPAINEN J: Endothelial function in long-term survivors of childhood acute lymphoblastic leukemia: effects of home-based exercise program. Pediatr Blood Cancer 60: 1546-1551, 2013.

JEHLICKA P, STOZICKY F, MAYER O JR, VARVAROVSKA J, RACEK J, TREFIL L, SIALA K: Asymmetric dimethylarginine and the effect of folate substitution in children with familial hypercholesterolemia and diabetes mellitus type 1. Physiol Res 58: 179-184, 2009.

JEHLICKA P, HUML M, SCHWARZ J, TREFIL L, KOBR J, SYKORA J: Reactive hyperaemia index as a marker of endothelial dysfunction in children with Crohn's disease is significantly lower than healthy controls. Acta Pediatrica 103: 55-60, 2014.

KADA-LOTTICK NS, ZELTZER LK, LIU Q, YASUI Y, ELLENBERG L, GIOIA G, ROBISON LL, KRULL KR: Neurocognitive functioning in adults survivors of childhood non-central nervous system cancers. $J$ Natl Cancer Inst 102: 881-893, 2010.

KELLY AS, MARLATT AL, STEINBERGER L, DENGEL DR: Younger age is associated with lower reactive hyperemic index but not lower flow-mediated dilation among children and adolescents. Atherosclerosis 234 : 410-414, 2014.

LEGER K, SLONE T, LEMLER M, LEONARD D, COCHRAN C, BOWMANPW, BASHORE L, WINICK N: Subclinical cardiotoxicity in childhood cancer survivors exposed to very low dose anthracycline therapy. Pediatr Blood Cancer 62: 123-127, 2015.

MODY R, LI S, DOVER DC, SALLAN S, LEISENRING W, OEFFINGER KC, YASUI Y, ROBISON LL, NEGLIA JP: Twenty-five-year follow-up among survivors of childhood acute lymphoblastic leukemia: report from the Childhood Survivor Study. Blood 111: 5515-5523, 2008. 
MOERLAND M, KALES AJ, SCHRIER L, VAN DONGEN MG, BRADNOCK D, BURGGRAAF J: Evaluation of the EndoPAT as a tool to assess endothelial function. Int J Vasc Med 2012: 904141, 2012.

MORI-KAWABE M, YASUDA Y, ITO M, MATSUO S: Reduction of NO-mediated relaxing effects in the thoracic aorta in an experimental chronic kidney disease mouse model. $J$ Atheroscler Thromb 22: 845-853, 2015.

MORISSON KM, DYAL L, CONNER W, HELDEN E, NEWKIRK L, YUSUF S, LONN E: Cardiovascular risk factors and non-invasive assessment of subclinical atherosclerosis in youth. Atherosclerosis 208: 501-505, 2010.

NATHAN PC, WASILEWSKI-MASKER K, JANZEN LA: Long-term outcomes in survivors of childhood acute lymphoblastic leukemia. Hematol Oncol Clin North Am 5: 1065-1082, 2005.

OEFFINGER KC, MERTENS AC, SKLAR CA, KAWASHIMA T, HUDSON MM, MEADOWS AT, FRIEDMAN DL, NEYSSA M, HOBBIE W, KADAN-LOTTICK NS, SCHWARTZ CL, LEISENRING W, ROBISON LL: Chronic health conditions in adult survivors of childhood cancer. $N$ Engl J Med 355: 1572-1582, 2006.

OSIKA W, MONTGOMERY SM, DANGARDT F, WÄHRBORG P, GAN LM, TIDEMAN EA: Anger, depression and anxiety associated with endothelial function in childhood and adolescence. Arch Dis Child 96: 38-43, 2011.

SELAMET TIERNEY ES, NEWBURGER JW, GAUVREAU K, GEVA J, COOGAN E, COLAN SD, DE FERRANTI SD: Endothelial pulse amplitude testing: feasibility and reproducibility in adolescents. $J$ Pediatr 154: 901-905, 2009.

SILVERMAN LB: Balancing cure and long-term risk in acute lymphoblastic leukemia. Hematology Am Soc Hematol Educ Program 2014: 190-197, 2014.

TOUBOUL PJ, GROBBEE DE, DEN RUIJTER H: Assessment of subclinical atherosclerosis by carotid intima media thickness: technical issues. Eur J Prev Cardiol 19: 18-24, 2012.

VAN DALEN EC, CARON HN, DICKINSON HO, KREMER LC: Cardioprotective interventions for cancer patients receiving anthracyclines. Cochrane Database Syst Rev 15: CD003917, 2011.

WIDLANSKY ME, GOKCE N, KEANEY JF JR, VITA JA: The clinical implications of endotehlial dysfunction. $J$ Am Coll Cardiol 42: 1149-1160, 2003.

WILSON PWF, PENCINA M, JACQUES P, SELHUB J, D'AGOSTINO R, O'DONNELL CJ: C-reactive protein and reclassification of cardiovascular risk in the Framingham Heart Study. Circ Cardiovasc Qual Outcomes 1: 92-97, 2008.

WOO JJ, DUK YC, IN-SANG J: Vascular endothelial dysfunction after anthracyclines treatment in children with acute lymphoblastic leukemia. Korean J Pediatr 56: 130-134, 2013. 